\title{
Sull'uso di una nuova congiunzione: due barra tre annotazioni
}

\author{
Barbara Patella
}

PUBBLICATO: 14 FEBBRAIO 2020

\section{Quesito:}

I lettori ci sottopongono due quesiti a proposito di barra: l'uno riguardante l'incertezza terminologica fra barra e sbarra favorita dalla somiglianza fonetica dei due vocaboli; l'altro, invece, relativo al nuovo uso di barra in qualità di congiunzione disgiuntiva in luogo di o, oppure, ovvero.

\section{Sull'uso di una nuova congiunzione: due barra tre annotazioni}

- econdo i dizionari, il termine barra, nei testi scritti, indica propriamente il segno grafico, costituito da una linea obliqua tracciata da sinistra a destra a partire dal basso $(/)$ - o piu di rado verticalmente $(\mid)$ - che è adoperato con varie funzioni:

- espungere quanto scritto;

- designare abbreviazione;

- segnalare la fine di verso di un testo poetico (o di una riga di un testo epigrafico) quando non si va a capo;

- indicare un'alternativa o un'opposizione fra due o più elementi (che possono essere lettere, parole, sintagmi o periodi veri e propri, ad esempio negli eserciziari scolastici o nei moduli da compilare).

È inoltre impiegato come:

- segno per la congiunzione copulativo-disgiuntiva e/o;

- segno di separazione (nelle frazioni, nelle date, nel simbolo di per cento e di per mille, in fonetica, nel linguaggio informatico ecc.).

Per i maggiori dizionari contemporanei (GRADIT, Sabatini-Coletti 2006, Zingarelli 20r9, Vocabolario Treccani online), col significato di 'segno grafico', sbarra è sinonimo di barra, parola da cui deriva con l'aggiunta del prefisso intensivo s-rispetto alla quale può denotare un tratto tracciato in qualunque verso (obliquo, verticale, orizzontale).

Anche sbarretta (o, meno comune, barretta) può essere utilizzato in luogo di barra o di sbarra: a tal proposito Giuseppe Antonelli, occupandosi della punteggiatura dall'Ottocento fino agli anni Duemila, in merito ai segni nuovi e virtuali, parla proprio di un "largo uso delle sbarrette (slash e backslash $<>>$ )" (Storia della punteggiatura in Europa, a cura di B. Mortara Garavelli, Roma-Bari, Laterza, 2008, p. 208). Quanto all'anglicismo backslash ('lineetta obliqua tracciata da sinistra a destra a partire dall'alto'), termine dell'informatica accolto dalla lessicografia italiana (GRADIT, Garzanti 2003, GDLI Supplemento 2004, Zingarelli 2004, Devoto-Oli 2004-2005), ci limitiamo a segnalare i calchi più diffusi, quali barra inversa (cfr. Zingarelli 2019, s.v. barra e s.v. backslash) e barra rovesciata (cfr. Roberto Lesina, Software \& Hardware. Dizionario dei termini informatici: italiano-inglese, inglese-italiano, Bologna, Zanichelli, I99I, s.v. barra rovesciata e s.v. backslash).

Si può stabilire, dunque, che barra, sbarra, sbarretta e barretta sono termini sinonimici ogniqualvolta designino il segno grafico ' $\%$. 
Spostiamo adesso l'attenzione sul processo di grammaticalizzazione in sincronia, in base al quale un elemento lessicale - nel nostro caso barra - assume anche funzione grammaticale.

A partire dall'edizione del 20I8, accanto alla definizione di barra quale 'segno grafico costituito da una lineetta [...]', il Devoto-Oli zor 8 - unico fra i dizionari dell'uso contemporaneo - segnala un altro uso, marcato come "colloquiale", secondo cui barra "tra due nomi o aggettivi indica che il loro uso può essere intercambiabile', e riporta il seguente esempio: "un attore/regista (si legge 'un attore barra regista'), una persona che può essere definita sia attore che regista". La crescente diffusione nel segno grafico nei testi scritti (sia cartacei sia in rete), con sconfinamenti anche nel parlato, è stata segnalata di recente anche da Paolo D'Achille, L'italiano contemporaneo, IV ed., Bologna, il Mulino, 2019, p. 2I3; inoltre, è in corso di stampa un articolo sullo sviluppo di barra come connettivo (cfr. Fiorentini, I., Miola, E., Disjunctive/conjunctive/whatever: the development of Italian barra ('slash') as a nonexhaustive connective, Language Sciences).

L'uso di barra con funzione grammaticale analoga alla congiunzione correlativa sia ... sia è ampiamente attestato nelle pagine di blog, di forum e di libri (perfino in formula reiterata) così come nell'italiano del piccolo e del grande schermo:

[...] e per di più durante la festa di compleanno di un modello barra opinionista televisivo, barra fashion blogger, barra attore di fiction? (Anna Chiatto, Ti amo ma niente di serio, Milano, Edizioni Piemme, 2016)

Ma la borsista a cui è stato proposto questo contratto aveva un rapporto sentimentale barra sessuale con lei? (da un'intervista di Danilo Lupo, "Non è l'arena", puntata del 9/6/19 trasmessa su La7)

Comincio con un bel periodo di prova barra apprendistato non retribuito di dodici, diciotto mesi e poi si vedrà. (dal film Smetto quando voglio, 20I4)

In verità, da oltre un decennio - almeno dal 2004 secondo le nostre ricerche -, la lingua italiana ha assistito a un ulteriore sviluppo della parola: sia nello scritto sia nel parlato, infatti, è in circolazione un secondo uso grammaticalizzato di barra (o, più raramente, sbarra), adoperato in alternativa alla congiunzione disgiuntiva $o$. Sotto forma di congiunzione, barra si rintraccia soprattutto in contesti con riferimenti temporali, in cui si può sottintendere una locuzione avverbiale approssimativa del tipo "all'incirca", "più o meno": arriverò a Roma fra due barra tre ore (= allincirca fra due o tre ore); per la spedizione ci vogliono quattro barra cinque giorni lavorativi (= piu o meno quattro, cinque giorni lavorativi); ci vediamo tra due barra tre mesi.

Nella sua nuova funzione grammaticale, barra è accolta in una vasta gamma di testi, quali blog, forum, romanzi, riviste e quotidiani, commenti e recensioni di clienti o acquirenti. Ecco alcuni esempi:

Per chi non avesse capito bene, i dieci barra dodici individui più ricchi d'Italia hanno risorse pari a quelle di 3 milioni e mezzo di poveri. (Alberto Statera, Il club degli straricchi e le mire di Visco e Soru, "la Repubblica", sez. Affari Finanza, p. I0, 25/9/2006)

Se solo li vendi a quelli che fanno i mercati rionali puoi ricavarci almeno due barra tre volte il centesimo che investi su ogni singolo pezzo. Fai conto che tu li vieni a pagare uno e in commercio viaggiano sui venticinque barra trenta centesimi al pezzo. (Andrea Begnini, Exit strategy, Round Robin Editrice, 2012)

La pentola più grande in possesso del nostro laboratorio contiene numero cinque litri d'acqua che tradotto in termini pratici sono circa tre barra quattro chili di frutta fatta a pezzi... (Marco, Confetture: la ricetta segreta di Donna Gnora, Blog di donnagnora.it, https://donnagnora.it/ 13/3/2017) 
Si contano, per giunta, grafie in cui barra è separato dai termini fra cui è interposto tramite virgola (scelta superflua giacché la virgola, da sola, basterebbe a conferire valore disgiuntivo) o trattino (scelta antieconomica se paragonata alle soluzioni ben più sintetiche che permettono di servirsi di un singolo carattere, quali o oppure /):

"Diciamo che ci sono, secondo un calcolo approssimativo che ti faccio ora a mente, almeno il settanta-barra-settantacinque per cento di possibilità che tu domani sarai interrogata in italiano...". (Laura Del Fiore, Innamorate pazze, Milano, Rizzoli, 2010)

Si può obiettare: ma il dominio vetteliano è tracimante e ora c'è l'effetto noia, vince solo lui, tre, barra quattro mondiali di fila sono troppi. (Benny Casadei Lucchi, Vettel, com'e triste dominare il mondo, "Il Giornale", sez. Sport, 24/9/2013)

Siamo noiosi, diciamolo, complice l'abitudine e la scarsa voglia di rischiare e così, prudentemente, beviamo le solite cinque - barra - sei zone vinicole. (Antonio Tomacelli, Zone vinicole dimenticate e dove trovarle, intravino.com, I/8/2018)

Fra gli esempi, si può notare anche una funzione equivalente a quella della preposizione tra quando indica approssimazione ("almeno il settanta-barra-settantacinque per cento" equivale ad "almeno tra il settanta e il settantancinque per cento"); infine barra, seppur sporadicamente, può dar luogo a un complemento di tempo (in frasi come saró a casa alle nove barra dieci per dire saró a casa tra le nove e le dieci).

Riguardo all'origine del fenomeno, possiamo ipotizzare che il passaggio da segno grafico (/) a segno verbale (barra) sia avvenuto per la concomitanza di due fattori, uno linguistico e uno extralinguistico: da un lato, l'influsso dell'inglese slash ('barra'), usato come congiunzione oltre che come sostantivo; dall'altro, la ritualità con cui il segno grafico "/" compare in testi di vario genere (amministrativo, didattico, saggistico, narrativo).

Quanto al caso parallelo di slash, adottato nella lingua inglese come congiunzione già dalla seconda metà del Novecento, vediamo come alcuni dizionari monolingui attestino tale funzione grammaticale: ad esempio Lexico, che nasce da una collaborazione tra Oxford University Press e Dictionary.com, registra slash come congiunzione di registro informale 'usata per legare alternative o parole che denotano o descrivono una duplice (o molteplice) funzione o natura' (cfr. Lexico.com, s.v. slash); così pure il Cambridge Dictionary online riporta l'uso parlato di slash, adoperato in luogo di or in frasi come "the room functions as a guest bedroom slash study" (cfr. Cambridge Dictionary, s.v. slash). E plausibile che a veicolare l'uso inglese siano state - e continuino a essere - in primis traduzioni da testi inglesi contenenti la congiunzione slash, nonché programmi tv, serie televisive e film che dalla lingua originale vengono doppiati in italiano.

Riguardo al secondo fattore, invece, la fortuna dell'uso di barra in qualità di congiunzione è in parte imputabile a un'inflazionata iconicità nei testi scritti, ossia alla tendenza odierna a inserire, oltre ai canonici segni di punteggiatura, una serie di segni grafici che possono essere sciolti e riprodotti oralmente fino a sedimentarsi nell'uso: come per punto, tra parentesi, fra virgolette, così è avvenuto per barra (si pensi, ad esempio, alle esigenze scaturite dal dettato o dalle letture in ambito scolastico, o semplicemente ai giudizi intermedi assegnati dagli insegnanti del tipo $7 / 7^{1 / 2}$ che hanno contribuito a una riproduzione "letterale" del segno "/": cosi il segno grafico, contenuto in numerosi testi, ha cominciato a essere restituito fedelmente a livello orale conbarrae non con la tradizionale congiunzione disgiuntiva o, oppure). A ben vedere, quindi, il ruolo sintetico che il segno grafico assume nella scrittura sortisce un effetto opposto nel suo esito orale. 


\section{Testimonianze di barra nell'italiano parlato}

Sul piano dell'oralità siamo in grado di testimoniare il fenomeno di grammaticalizzazione di barra in trascrizioni di interviste e di udienze, in resoconti di sedute di consigli comunali, in conferenze stampa e convegni, come pure in doppiaggi di programmi televisivi e in copioni cinematografici. Un caso di parlato spontaneo, ad esempio, è contenuto nella trascrizione di unintervista condotta dall'Università di Roma in occasione di una ricerca sui percorsi di studio all'estero:

R: Ehm scritti! Cioè nel senso a risposta aperta la parte... poi ovviamente la parte orale era il progetto, la tesina era di quindici barra venti pagine poi... la presentavi in dieci minuti (Guido Benvenuto, Percorsi di studio Erasmus alluniversitá, Edizioni Nuova Cultura, 2015, p. 179, Appendice C.2: Trascrizione interviste agli studenti Erasmus di area psicologica).

Oltre al parlato trascritto, offrono preziose testimonianze orali molti video diffusi in rete (amatoriali e non): dalle recensioni di prodotti tecnologici ("solamente con notifiche attive e utilizzo smart dell'orologio sono riuscito a fare anche due barra tre settimane": youtube.com) alle conferenze stampa calcistiche ("c'è solo l'attesa di questa prima partita, ma la prima partita è sempre così, perché ritorni dopo due barra tre mesi di sosta”: vecchiasignora.com), fino ad arrivare ai convegni istituzionali, come il convegno Dalla storia...verso il futuro. Stati Generali dei Consulenti del Lavoro, tenutosi a Roma l'iı gennaio 2019, in cui il Presidente del Consiglio Giuseppe Conte ha dichiarato che "nel 2org per un lavoratore che andrà in pensione con questa riforma ne verranno occupati due barra tre" (radioradicale.it). Proprio il sito di Radio Radicale mette a disposizione numerose trascrizioni di documenti audiovisivi (registrazioni di convegni, dibattiti, conferenze, assemblee, congressi), che consentono di rilevare la vitalità di barra congiunzione in ambito politico e in ambito economico:

[...] ma è ancora più grave perché poi Camera e Senato nominano tutte le autorità indipendenti, quindi a cascata diventa un sistema in cui due barra tre persone sostanzialmente sono i capofila di tutto quello che succede dopo. (discorso di Emma Bonino tratto dalla registrazione video del dibattito Elezioni regionali 2010: videowebchat con Emma Bonino per il lancio di listaboninopannella.it e della webtv, Roma, I/2/20IO, radioradicale.it)

Oggi il processo amministrativo che porta alla realizzazione di un nuovo edificio comporta mediamente uno barra due anni di procedura amministrativa e a seconda delle difficoltà tecniche un anno e mezzo barra tre anni di realizzazione. (discorso di Marco Dettori tratto dalla registrazione video del convegno Presentazione del Libro bianco sulla fiscalità immobiliare, Roma, 26/9/2018, radioradicale.it)

In apertura del paragrafo si è accennato alla presenza di barra congiunzione in luogo di o, oppure anche nell'italiano del cinema; ecco un dialogo tratto da un film di Enrico Oldoini e recitato da Claudio Bisio e Angela Finocchiaro:

Dottoressa: No, rapporti nel senso rapporti sessuali, frequenza. Deh, non esageri!

Giancarlo: No, no, uno barra due volte alla settimana, scriva!

(dal film I mostri oggi, 2009)

Come spesso accade, le mode e le innovazioni linguistiche sono suscettibili di riflessioni metalinguistiche e di giudizi non sempre positivi nella percezione dei parlanti, difatti la frequenza con cui barra, in veste di congiunzione, si sia imposta nell'uso di parlanti e scriventi non ha risparmiato critiche al riguardo: 
"Mi sorprendi, lo sai? Non pensavo che anche tu usassi il termine must. Linguaggio molto giovanilistico, direi. Magari adoperi anche frasi tipo sette barra otto oppure muovi le due dita verso l'alto ad indicare una frase tra le virgolette?». (Marco Di Tillo, Omicidio all'acquario di Genova. La nuova indagine dell'ispettore Marco Canepa, Genova, Fratelli Frilli Editori, 2019)

Ed ecco che ci siamo: la "barra". Ogni volta che sento qualcuno che fa un lavoro con "barra", mi fermo. Niente di buono arriva mai dopo una barra. Significa o che non può permettersi di vivere con una sola entrata o che non sa decidere cosa vuole fare da grande. Un dentista barra DJ; un contabile barra danzatore del ventre; un intermediario finanziario barra barista... (Erin Brady, Un ultimo appuntamento al buio, trad. di Elisa Ponassi, Triskell Edizioni, 2019)

\section{Cita come:}

Barbara Patella, Sull'uso di una nuova congiunzione: due barra tre annotazioni, "Italiano digitale", 2020, XII, 2020/1 (gennaio-marzo)

DOI: $10.35948 / 2532-9006 / 2020.3230$

\section{Copyright 2020 Accademia della Crusca}

Pubblicato con licenza creative commons CC BY-NC-ND 